\title{
Exercise for Brain Health: An Investigation into the Underlying Mechanisms Guided by Dose
}

\author{
Danylo F. Cabral ${ }^{1,2}$. Jordyn Rice ${ }^{1,2}$ - Timothy P. Morris ${ }^{3} \cdot$ Tatjana Rundek $^{2,4}$ - Alvaro Pascual-Leone ${ }^{3}$. \\ Joyce Gomes-Osman ${ }^{1,2,3,4}$ (iD
}

Published online: 13 June 2019

(C) The American Society for Experimental NeuroTherapeutics, Inc. 2019

\begin{abstract}
There is a strong link between the practice of regular physical exercise and maintenance of cognitive brain health. Animal and human studies have shown that exercise exerts positive effects on cognition through a variety of mechanisms, such as changes in brain volume and connectivity, cerebral perfusion, synaptic plasticity, neurogenesis, and regulation of trophic factors. However, much of this data has been conducted in young humans and animals, raising questions regarding the generalizability of these findings to aging adults. Furthermore, it is not clear at which doses these effects might take place, and if effects would differ with varying exercise modes (such as aerobic, resistance training, combinations, or other). The purpose of this review is to summarize the evidence on the effects of exercise interventions on various mechanisms believed to support cognitive improvements: cerebral perfusion, synaptic neuroplasticity, brain volume and connectivity, neurogenesis, and regulation of trophic factors. We synthesized the findings according to exposure to exercise (short- [1 day-16 weeks], medium- [24-40 weeks], and long-term exercise [52 weeks and beyond]) and have limited our discussion of dose effects to studies in aging adults and aged animals (when human data was not available).
\end{abstract}

Key Words Physical exercise $\cdot$ cognitive brain health $\cdot$ exercise dose $\cdot$ aging brain $\cdot$ older adults $\cdot$ physiological mechanisms

\section{Introduction}

Adding to the well-established benefits of physical exercise for decreasing mortality and morbidity and improving physical function, there has been a wealth of evidence generated in

Danylo F. Cabral and Jordyn Rice contributed equally to this work.

Joyce Gomes-Osman

j.gomes@miami.edu

1 Department of Physical Therapy, University of Miami Miller School of Medicine, 5915 Ponce de Leon Boulevard, 5th Floor, Coral Gables, Florida 33146, USA

2 Evelyn McKnight Brain Institute, University of Miami Miller School of Medicine, 1150 Northwest 14th Street, Suite 309, Miami, Florida 33136, USA

3 Berenson-Allen Center for Noninvasive Brain Stimulation and Division of Cognitive Neurology, Department of Neurology, Beth Israel Deaconess Medical Center, Harvard Medical School, 330 Brookline Avenue, Boston, Massachusetts 02215, USA

4 Department of Neurology, University of Miami Miller School of Medicine, 1150 Northwest 14th Street, Suite 309, Miami, Florida 33136, USA recent years to support a link between the practice of regular exercise and cognitive brain health in older adults. The wealth of data that supports improved cognition following the practice of regular exercise is particularly relevant given that older adults (i.e., individuals aged 65 and older) represent the fastest growing demographic worldwide. In approximately 10 years, the older population will outnumber children for the first time in United States history, reaching $20 \%$ of the overall population [1]. The maintenance of cognitive brain health is consistently cited as the top health-related concern for aging adults and a key factor for the maintenance of maximum autonomy and independence [2].

Age-related cognitive impairment mostly affects the speed of processing of mental tasks and tasks that require memory and executive functioning (which covers a broad spectrum of abilities, but in short, refers to tasks that require organizing, planning, reasoning, and problem solving) [2]. These agerelated cognitive impairments largely reflect brain atrophy, most pronounced in the frontal lobe, resulting in decreased gray matter in critical areas involved in higher-order cognitive processes, such as the prefrontal, temporal lobes, and hippocampus $[3,4]$. In addition, impaired conduction of neural signals arising from lesions to white matter tracts [5] and an 
unfavorable neurochemical environment caused by decreased neurotrophins and trophic factors [6], neurotransmitter imbalances $[7,8]$, and deposition of toxic protein aggregates such as amyloid beta $(\mathrm{A} \beta)$ all contribute to increased free radicals and impaired neuronal function [9].

It is encouraging that adopting a lifestyle change in the form of the practice of regular exercise constitutes an opportunity for action to promote healthy cognitive aging, but in order for exercise to be used as a "medicine" [10], it is necessary to become much more specific with the dose. Most recently, the Physical Activity Guidelines for Americans [11] was updated and mentioned the importance of exercise for brain health and emphasized generally moving more and sitting less. However, within the literature, there is great heterogeneity in the methodologies and findings across studies regarding exercise dose, which have only now begun to be addressed systematically. For example, there is no consensus on the optimal doses or mode of physical exercise to maximize cognitive benefits, but a recent large-scale systematic review and regression found that the total time spent exercising ( $\sim 52 \mathrm{~h})$ was the only significant correlate of improved cognition. Additionally, the same study found that most consistent improvements from physical exercise interventions occur in executive functions and processing speed, an encouraging finding given that these are among the cognitive domains that first begin to show age-related cognitive decline [12].

Although this initial examination of dose is a definite positive step in the right direction, a large proportion of the findings regarding the structural and molecular effects of exercise (hypothesized to drive cognitive improvements) have been discovered in rodent studies, and the generalizability of these results to humans is unclear. The neurobiological underpinnings linked to exercise-mediated cognitive improvements include the ability to counteract the age-related atrophy of gray and white matter [13-17]; increase the vasculature, dendritic spine density, and complexity of the hippocampus; enhance synaptic plasticity [18]; and increase the release of fundamental biochemical mediators of neuronal survival (i.e., neurotrophins, trophic factors) [19-22]. Pertinently however, it is not clear at which dose these effects take place and with which exercise modes. Adding to the complexity, fewer of the studies in animal models have been performed in aging animals, leading to questions in the generalizability to the aging process itself.

Therefore, the purpose of this present study is to summarize the evidence of the effects of participation in an interventional exercise research study on the mechanisms believed to support cognitive improvements in the aging brain: cerebral perfusion, synaptic neuroplasticity, brain volume and connectivity, neurogenesis, and regulation of trophic factors [23-26]. In addition, this study aims to synthesize these effects in function of the dose (short-, medium-, and long-term exercise). For the purposes of this review, we considered short-term exercise to be 1 day to 16 weeks, medium to be 24 to 40 weeks, and longterm to be 52 weeks and beyond. In addition, we have limited our discussion of dose effects to studies in cognitively healthy individuals at least 50 years of age and aged rodents of at least 18 months (when human data was not available). Given the effects of exercise on cognitive function domains have been addressed elsewhere [12], we herein describe cognitive functions per their broad cognitive abilities and where possible adhering to the Cattell-Horn-Carroll theory of human cognitive abilities [27].

The majority of the included articles were found in the medical database MEDLINE/PubMed and through additional manual search in reference lists of included studies and expert knowledge of relevant papers. The search strategy included terms related to the intervention (exercise), participants (older adults, aged rodents), and the main physiological outcomes hypothesized to underlie cognitive improvements in the aging brain (cerebral perfusion, synaptic neuroplasticity, brain structure, neurogenesis and synaptogenesis, and trophic factors). Searches were conducted from May 2018 to February of 2019. The complete details of the inclusion criteria and search strategy are presented as Supplemental Material.

\section{Cerebral Perfusion}

One of the mechanisms shown to support cognition following regular exercise is the maintenance and improvement of cerebral blood flow. There is a decrease of approximately $30 \%$ in global cerebral blood flow from midlife to older age [28], which has been linked to age-related atrophy and decreased metabolism [29].

Physical exercise is undoubtedly associated with not only increased cardiac output but also a redistribution of the total blood volume to meet the increased partial pressure of arterial carbon dioxide $\left(\mathrm{PaCO}_{2}\right)$ and the resultant increased demand of the peripheral vasculature supplying the exercising muscles. The cerebral vasculature undergoes changes as well, albeit less pronounced. The elevation in arterial cerebral blood flow seems to be less dramatic, showing progressive increases in intensities up to $60 \% \mathrm{VO}_{2} \max$, reaching a plateau, and then returning to resting levels with further increases in exercise intensities [30].

In this context, exercise-mediated increases in middle cerebral artery velocity (MCAv) enable the probing of the mechanism of cerebral homeostasis. Table 1 summarizes the results regarding cerebral perfusion in the aging brain. Studies have demonstrated smaller MCAv increases at rest and during exercise but did not report differences in the proportion of exercise-mediated increase in MCAv between young and older adults $[31,32]$. The age-dependent decrease in MCAv could represent a physiologic compensatory mechanism to counteract the elevation in blood pressure and global 


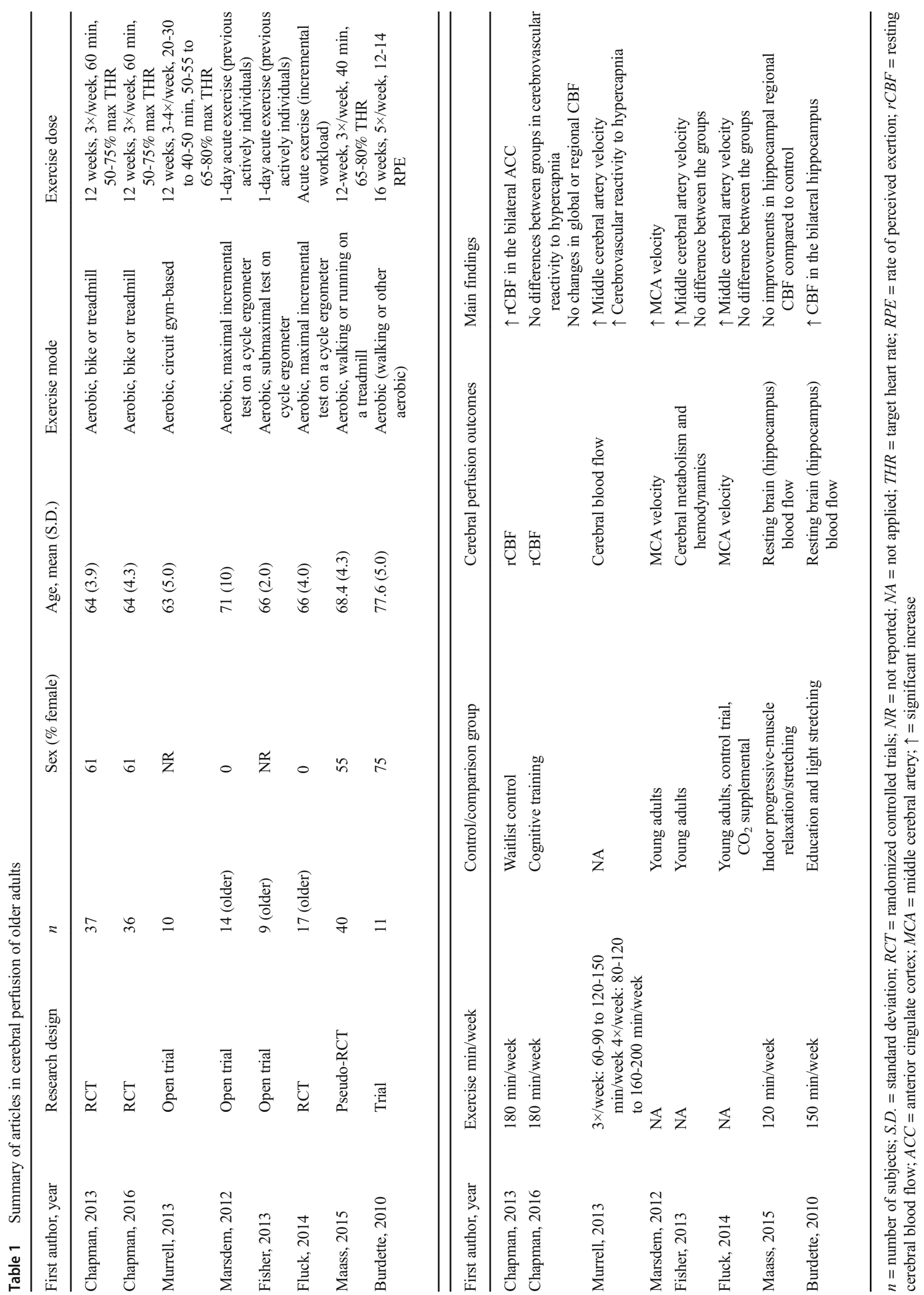


decreased elasticity of blood vessels in older age. In addition, improvement in cerebrovascular reactivity to hypercapnia (i.e., cerebrovascular reactivity or the ability of blood vessels to vasodilate) associated with exposure to short-term (i.e., 12 weeks) moderate- to high-intensity aerobic exercise has been found to be similar between young and older adults [33].

Studies have examined the effect of short-term (ranging from 12-16 weeks) moderate-intensity aerobic exercise on regional or whole-brain cerebral perfusion and have found mixed results. Following one 12-week intervention, there was increased cerebral blood flow (CBF) in the bilateral anterior cingulate cortex (ACC) when compared with a waitlist control [34], but a subsequent study utilizing the same exercise dose found no changes in global or regional CBF when making comparisons with a cognitive training group [35]. One 12-week intervention was not sufficient to improve hippocampal regional CBF, when compared with muscle relaxation/stretching [17], but a 16-week intervention in an older sample ( $\sim+10$ years) who had subjective memory complaints found improvements in bilateral hippocampal CBF when compared with a comparison group of education and light stretching [36].

\section{Synaptic Neuroplasticity}

Synaptic neuroplasticity is defined as long-lasting changes in efficacy of synaptic connections (i.e., long-term potentiation (LTP) or long-term depression (LTD). Synaptic neuroplasticity was first described following repetitive electrical stimulation of hippocampal neurons, and when measured with electrodes placed directly in hippocampal tissue, evidence for the role of LTP and LTD in memory consolidation was shown [37, 38]. LTP is currently the strongest candidate to explain brain-wide synaptic activity implicated in cognitive processes and learning (both cognitive and motor), and there is a documented decreased LTP associated with aging in experimental rodent models $[18,39]$. In experimental studies with young rodent models, robust improvement in visuospatial ability after regular exercise is observed and correlated with enhanced LTP of synaptic activity in hippocampal cells $[18,40]$. Furthermore, exercise rescues LTP ability and promotes cognitive gains in different young rodent models of impaired LTP [41-44].

Only 2 studies have examined the influence of exercise on LTP in aged rodent models and have found improved synaptic plasticity following exposure to long-term exercise interventions. Kumar et al. [45] assessed short-term exercise exposure in the form of a 12-week intervention and found increased LTP and improved performance on the cue discrimination task of the water Morris maze and object recognition memory. O'Callaghan et al. [46] assessed LTP in aged rats following long-term exercise exposure (8-month-long aerobic exercise intervention) initiated in middle age. The authors found that the long-term exercise prevented age-related decreases in LTP and spatial learning.

\section{Brain Structure}

\section{Volume}

The aging brain undergoes selective atrophy, mainly in the prefrontal cortices and medial temporal lobes [47, 48]. For instance, it has been reported that beginning at approximately age 50 , the hippocampus shrinks 1 to $2 \%$ every year in healthy older adults [47]. These decrements in volume have been linked to age-related decreases in memory and executive function $[49,50]$. Decreased age-related atrophy has historically been implicated in explaining exercisemediated improvements in cognitive performance for the older adult population [13].

Table 2 summarizes the results of studies that investigated the effects of exercise on brain volume. The 2 studies that examined the influence of short-term (12-week) moderateintensity aerobic interventions on brain structure failed to find differences in whole-brain gray matter volume, either when exercise was compared to a waitlist control [51] or a stretching/muscle relaxation comparison [17]. Although no between-group differences were found, a subanalysis in the Maass et al. [17] study demonstrated regional increases in the volume of the hippocampal head exclusively in those who exhibited improvements in fitness and hippocampal perfusion [17]. Taken together, these results suggest that perhaps certain individuals who show mediatory gains in secondary outcome measures (such as cardiorespiratory fitness and hippocampal perfusion) may also exhibit benefits in exercise-induced changes in brain structure.

Medium length interventions may have greater impact in the regional brain structure however. Kleemeyer et al. [52] found that 26 weeks (i.e., 6 months) of both low and high aerobic exercise was associated with increased neuron density and volume in the hippocampus, and these 2 outcomes were correlated [52]. Ruscheweyh et al. [15] found that both highintensity exercise in the form of Nordic walking and lowintensity exercise in the form of gymnastics improved gray matter volume, mainly in prefrontal and cingulate cortices, when compared with a no-intervention control [15]. In addition, a low- to moderate-intensity aerobic exercise was associated with increased regional brain volume in both gray and white matter areas relevant to the processing of attentional control and memory processes and shown to be implicated in age-related atrophy, when compared with stretching and toning [13]. Namely, the most prominent increases in volume were found in the ACC, right superior temporal gyrus, right middle frontal gyrus, and anterior white matter. However, a 


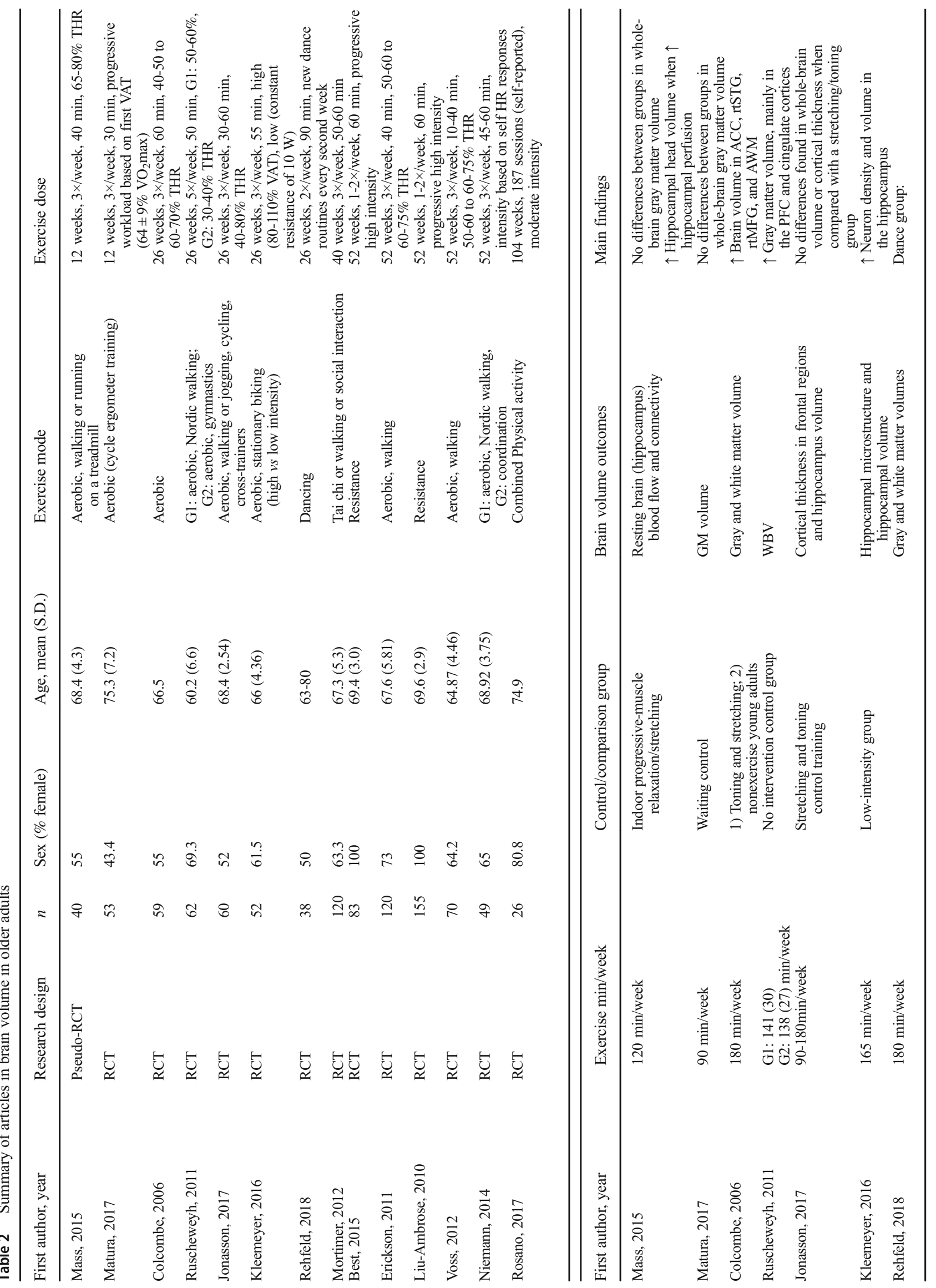




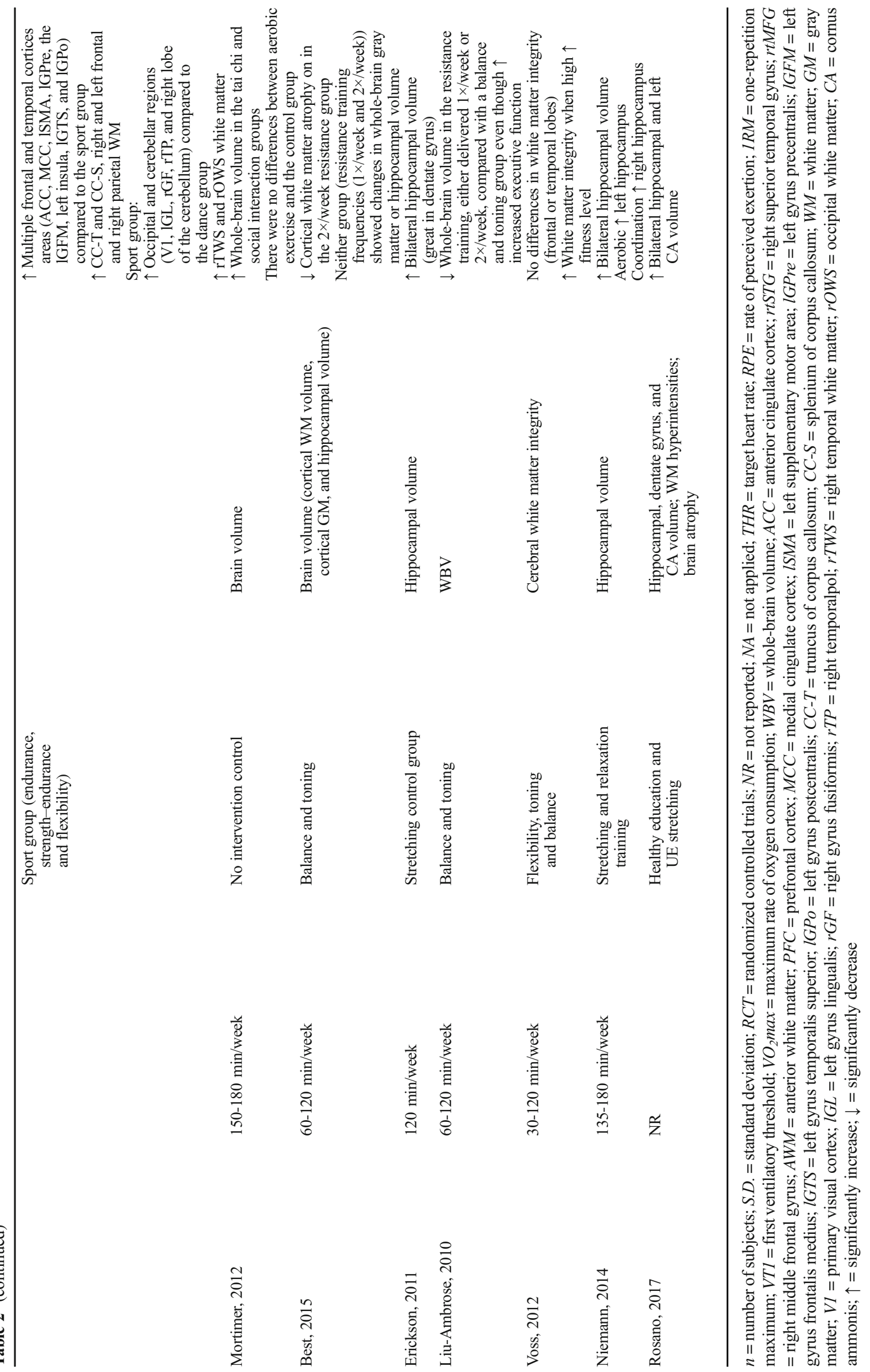


more recent intervention delivered with similar dose and exercise mode (differing in total time per week 90-180 vs $180 \mathrm{~min}$ ) did not find differences in frontal and hippocampus cortical thickness when compared with a stretching/toning group, despite an improvement in a composite cognitive score (episodic memory, processing speed, updating, taskswitching, visuospatial reasoning) in the aerobic exercise group [53]. The author discussed 2 possible reasons for these controversial findings, especially regarding brain volume. First, it is possible that an exercise intervention longer than 6 months may be required to achieve greater changes in brain volume. Second, the myriad cognitive improvements following a 6-month exercise intervention may be driven by other physiological processes, apart from macrostructural changes.

Furthermore, there is evidence suggesting differential structural effects according to exercise mode. For instance, individuals demonstrated increased brain volume in various frontal and temporal cortical areas following a 26-week dancing intervention, whereas individuals participating in a resistance training and flexibility group demonstrated increased brain volume in occipital and cerebellar regions [54]. Interestingly, both groups had similar increased aerobic fitness and small changes in attention and visuospatial memory. It was hypothesized that the constant novelty and greater demand in attention and memory posed by the choreography in the dancing group created a greater challenge than the repetitive routine in the resistance training and flexibility group, possibly accounting for the larger improvements in brain volume.

The only study we found proposing moderate-high exposure to exercise ( 40 weeks) incorporated 2 distinct nonaerobic modalities, tai chi and social interaction, and made comparisons to light aerobic exercise and a no-exercise control group [55]. Interestingly, only tai chi and social interaction were associated with increased whole-brain volume and improved processing speed and short-term memory. Contrary to previous findings in the literature, there were no differences between the light aerobic exercise and the control. We believe that this is possibly due to the fact that unlike the other studies that used light exercise interventions [15, 52], there was not a preferred intensity or a target zone, allowing the participants to walk at their self-selected pace. Although walking interventions are common in the literature, support for a potential floor effect in the aerobic exercise group comes from a subanalysis which revealed that faster walkers exhibited greater processing speed and short-term memory and lower brain tissue loss than slower walkers. Although there was no target intensity for the tai chi, this intervention requires a higher level of intellectual involvement and sustained attention compared to walking, which may have contributed to the results. Taken together, it is possible that aerobic exercise needs to be dosed at a minimum intensity and session time to achieve a threshold for structural gains, whereas other interventions such as tai chi, gymnastics, or social interaction may enlist distinct mechanisms due to the fact that they are more cognitively challenging.

The remainder of human studies examined high exercise exposure in yearlong interventions. Liu-Ambrose [56] assessed the effects of resistance training, either delivered $1 \times /$ week or $2 \times /$ week, and made comparisons with a balance and toning group delivered $2 \times /$ week. Interestingly, the authors found decreased whole-brain volume and increased executive function in the resistance groups when compared with the balance and toning group. The decreased whole-brain volume was unexpected but consistent with a mean of about $0.5 \%$ decreased annual whole-brain volume reported in healthy aging $[57,58]$. A latter follow-up study performed 1 year after intervention demonstrated that neither group showed changes in whole-brain gray matter or hippocampal volume, but the $2 \times /$ week did show reduced cortical white matter atrophy [59]. Additionally, both resistance training frequencies $(1 \times /$ week and $2 \times /$ week) maintained their increased processing speed and attention, but the $2 \times /$ week group additionally demonstrated improved short-term memory [59]. Thus, further research is needed to examine the relationship between structural brain changes and resistance training, especially given the improvements in certain cognitive abilities noted.

Both light to moderate aerobic exercise and a flexibility/ toning/balance interventions were found to be associated with similar levels of white matter integrity in the frontal and temporal lobes, executive control, and short-term memory [60]. A subanalysis revealed that individuals who made greater improvements in cardiovascular fitness made greater improvements in white matter integrity and short-term memory. An average increase in cardiovascular fitness of $8 \%$ following a moderate aerobic exercise intervention was associated with an average $2 \%$ increase in hippocampal volume, when compared with a yearlong stretching intervention [14]. Similar increases in hippocampal volume have been reported with a coordination intervention [61]. Interestingly however, aerobic exercise increased volume in the left hippocampus and the coordination group increased the volume of the right hippocampus. The authors attributed the differential effects to distinct demands posed by the 2 interventions; the right hippocampus is more highly engaged in spatial memory processes and, thus, theoretically more active during coordination exercises, whereas the left hippocampus is more engaged in verbal memory and highly associated with the increase in fitness level [62].

In the study proposing the highest exposure to exercise ( 2 years), a multimodal intervention consisting of moderateintensity physical activity (walking, lower extremity resistance training, balance, stretching, and behavioral counseling) was associated with improved bilateral hippocampal and left cornu ammonis volumes, when compared with a control group consisting of healthy education and stretching. In 
addition, the authors reported that greater self-reported adherence to the intervention was associated with greater hippocampal volume [63].

\section{Connectivity}

In addition to structural changes, age-related cognitive decline is associated with impaired functional activation of neural networks. For example, older individuals with age-related cognitive decline have exhibited impaired neural interhemispheric communication between the frontal and posterior cortices [64] and disrupted frontotemporal activation during memory-related tasks [65-67]. The studies described herein measure the degree of connectivity (i.e., degree of simultaneous cortical activation) between spatially distant cortical areas either during resting state (when the individual is not engaged in a specific thought or task) or during the performance of a specific task.

Table 3 summarizes the results of studies that investigated changes in brain connectivity after exercise intervention. Short-term exposure (12 weeks) to moderate-intensity exercise has been associated with a reduction in the number of cortical areas active during the performance of a memory semantic task, which was attributed to a potential increase in the efficiency of neural networks [68]. In addition, the same intervention delivered at a slightly higher dose (16 weeks) was associated with connectivity of the hippocampi and increased interconnectivity between the hippocampi and ACC, when compared with education and light stretching [36]. The interconnections with the ACC are relevant as this structure has reciprocal connections with the prefrontal cortex and is active during tasks that require executive control [69].

Medium-term exposure (6 months) to light to moderate aerobic interventions (6 months) was also associated with improved connectivity. The authors found decreased activation of the ACC, which was associated with improved executive control. The authors attributed the improved executive control to a task-related increase in various areas in the attentional circuitry network (middle frontal gyrus, superior frontal gyrus, and superior parietal lobules) which in theory decreased the demand of the ACC during the task [70]. The authors attributed these findings to an increase in cardiovascular fitness, not exhibited by the stretching/toning group. Taken together, these results suggest that a global increase in connectivity and increased focal connectivity in areas pertinent to executive control and memory are possible with approximately 3 to 6 months of moderate aerobic exercise.

Similar to these previously reported findings, long-term exposure to exercise was also shown to improve connectivity and executive control, but with differing patterns according to exercise mode [71]. Specifically, 1 year of light to moderate aerobic exercise was associated with an improvement in the connectivity between areas within the default mode network and within the frontal executive network. On the other hand, the stretching/toning/balance intervention was associated with increased connectivity within areas of the DMN at 6 months and within the frontoparietal network. However, neither group showed differences in task-switching ability or spatial working memory [71]. The default mode network, frontal executive network, and frontoparietal network are highly engaged during the performance of executive control and spatial memory tasks, and connectivity between these areas is impaired in age-related cognitive decline [72, 73].

Voelker-Rehage et al. [74] also found improved perceptual speed and executive functioning with different exercise modes and differential patterns of improved connectivity. The authors found that both moderate to high aerobic exercise and coordination: 1) improved neural efficiency during the performance of an executive control task and 2) improvements in executive attentional control and perceptual speed, albeit with differential patterns of connectivity. The aerobic group showed a decreased activation of left superior and middle frontal, bilateral medial frontal gyrus, the left ACC, the left parahippocampal gyrus, and the right superior and middle temporal gyrus. The coordination group showed increased activation of inferior frontal gyrus, superior parietal cortex, and thalamus and caudate. In addition to the differential connectivity patterns between the 2 intervention groups, the authors also found that the improved perceptual speed and executive functioning in the aerobic exercise was driven by an improvement in cardiovascular fitness. Taken together, these studies suggest that long-term exposure to different modes of exercise may be associated with certain cognitive benefits via distinct patterns of functional connectivity.

\section{Neurogenesis and Synaptogenesis}

Animal models enable the study of the microscopic changes in brain structure that support exercise-mediated cognitive benefits, such as the formation of new neurons (neurogenesis) and synapses (synaptogenesis) in the hippocampus. One of the strongest and earliest links between exercise and cognition was found on neurogenesis [75-77], which was 1 of the first hypotheses used to explain the neurobiological underpinnings of exercise-mediated cognitive performance benefits. However, when considering the generalizability of these microscopic findings to translational research in aging humans, it is pertinent to also consider how age might impact processes such as neurogenesis and synaptogenesis. For instance, older rodents ( 9 months) show hippocampal neurogenesis at half the rate of young adult rodents ( 6 weeks), and that by 24 months, the rate of neurogenesis is further decreased to $17 \%$ [78].

Despite the age-related decrease in hippocampal neurogenesis, it is encouraging that neurogenesis can be enhanced with exercise in aged rodents. For example, 1 month and half of voluntary wheel-running (VWR) was shown to 


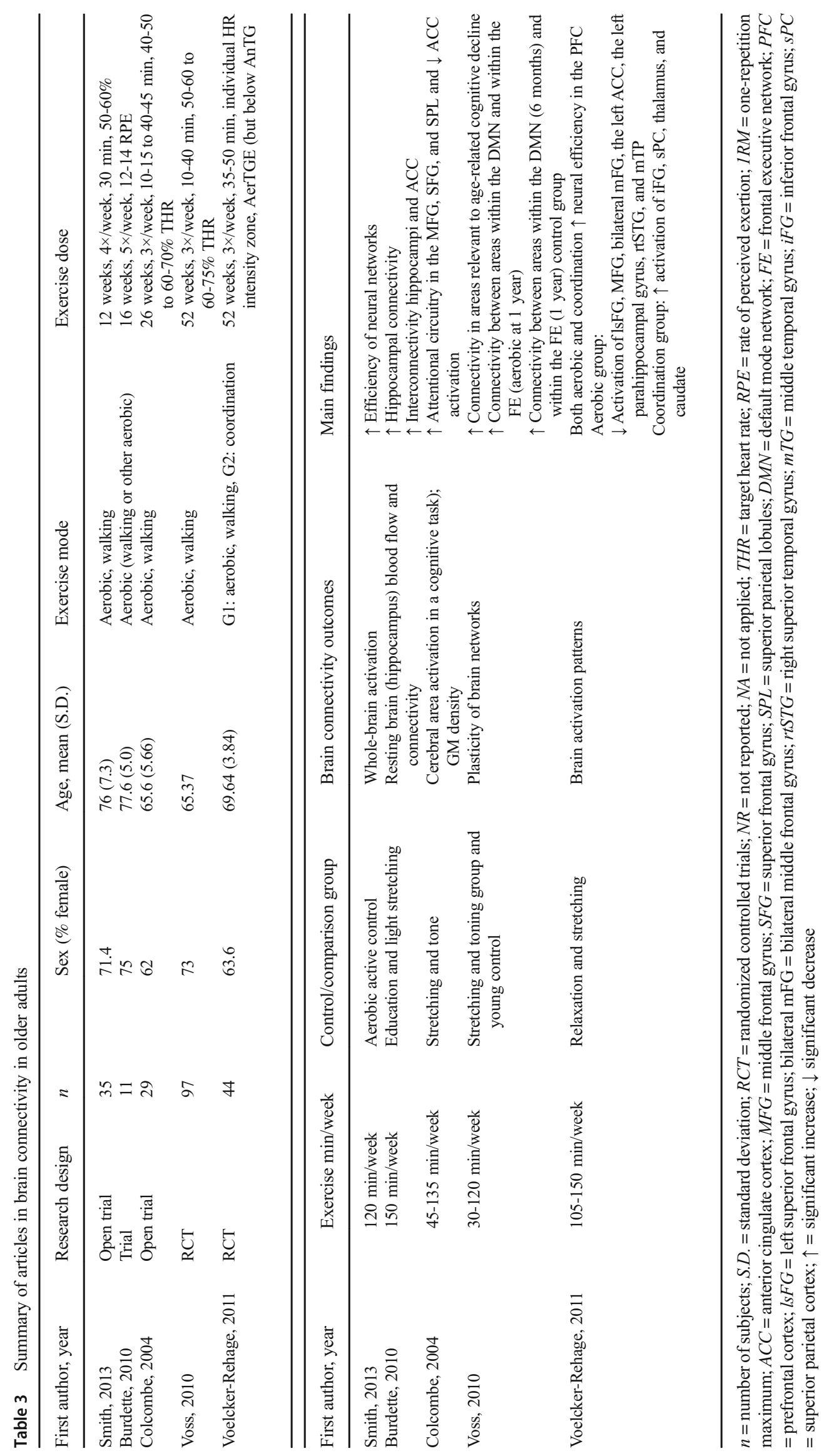


revert the decline in neurogenesis in 19-month-old rodents by $50 \%$ and increase gliogenesis by $20 \%$, an improvement that was paralleled with improved spatial learning, when compared with sedentary control rodents [79]. Interestingly, the authors found no difference in the number of new cells or in fine morphology between older exercised and young mice, suggesting that exercise may have enhanced the capacity for conversion of precursor cells into neurons. The aged exercised mice showed increased rate of conversion by 3 -fold when compared with the sedentary aged mice (25.6vs $9.5 \%$, respectively). Regarding the exercise dose necessary to induce such benefits, there is evidence to support that 10 to 28 days of VWR was sufficient to induce increases in precursor cell divisions associated with hippocampal neurogenesis [78, 80, 81]. However, 1 study found that 14 days of VWR in 22month-old mice was associated with lower neurogenesis [82].

Neural stem cells (NSC), considered to support the regenerative function of the brain, are decreased to about $70 \%$ in 18 month-old mice and 90\% in 24-month-old mice [83]. Interestingly, 21 days of VWR exercise attenuated the agedependent decrease in NSC proliferation in endogenous extrahippocampal areas by $67 \%$ in 18 -month-old mice but failed to do so in 24-month-old mice [83]. One study did find increased proliferation of hippocampal NSC in 24-month aged mice after 3 days of VWR [84]. Taken together, it is possible that aerobic exercise may have differential effects in different types of NSC.

Other studies have shown that exercise may influence neurogenesis through different pathways. For instance, evidence suggests that exercise rescues the levels of enzymes TET1 and TET2 (Ten-eleven translocation 1 and 2), shown to be decreased in aging animals and act to regulate hippocampal neurogenesis [85]. In addition, exercise has been shown to revert the toxic effect of lipopolysaccharide, a bacterial endotoxin shown to reduce hippocampal neurogenesis levels when expressed in 21-month-old mice [86]. Furthermore, exercise decreased new microglia, immune cells linked to low-grade neuroinflammation that may contribute to decreased plasticity and increased new neuron survival [87].

Similarly, to neurogenesis, synaptogenesis is also decreased in aged rodents. For example, the number of presynaptic receptors in the hippocampus is decreased [88]. Unfortunately, we could not find evidence of the impact of exercise in aged rodents, but studies suggest improvements in synaptogenesis in young exercised rodents. For instance, VWR is associated with increased synaptophysin, a marker of synaptogenesis in young rodents [89]. In addition, nonaerobic exercise (walking through an obstacle course) was also associated with new synapses in other areas of the brain, mostly related with the control of motor activity [90].

As mentioned previously, animal studies show that the capacity for neurogenesis can be positively influenced by exercise. However, the generalizability of decreased neurogenesis found in aged mice needs to be carefully interpreted when applied to older adults. A recent cross-sectional study in postmortem healthy individuals from 14 to 79 years found that adult hippocampal neurogenesis may persist throughout the life span, including the 8 th decade of life, despite the common clinical finding of age-related cognitive decline [91]. Neurogenesis (measured by levels of intermediate neural progenitors, immature neurons, glia, and mature granule in the dentate gyrus) was found to be at similar levels in individuals from 14 to 80 years of age. The authors attributed the aspects of preserved cognitive performance in older adults to the maintenance of those levels of neurogenesis. However, in the comparison between young adults and older adults, the authors did find decreased angiogenesis, neuroplasticity markers of PSA-NCAM+ (polysialylated neural cell adhesion molecule) cells of different morphologies, and capacity for neurogenesis (assessed by the number of quiescent NSC), potentially implicated in the aspects of cognition that show decreases in the aging brain [91].

\section{Trophic Factors}

Several trophic factors have been identified to support cognition in aging adults. The most commonly discussed factors that are supportive of cognition include brain-derived neurotrophic factor (BDNF), vascular endothelial growth factor (VEGF), and insulin-like growth factor-1 (IGF-1). These trophic factors work in concerted fashion to modulate exerciseinduced cognitive improvements [23]. IGF-1 is important for vascular maintenance and remodeling. Both IGF-1 and VEGF are thought to mediate neurogenesis and angiogenesis and influence the induction of hippocampal BDNF.

BDNF supports neuronal development and has been demonstrated to be crucial for exercise-related improvements in cognitive function. Blocking BDNF annuls the cognitive improvements induced by exercise [92], demonstrating its significance for cognitive change. Additionally, running increases IGF-1 and VEGF in the hippocampus [93, 94], both of which are crucial to exercise-induced plasticity and cognitive improvements. IGF-1 is increased in the hippocampus following exercise, and blocking IGF-1 receptors reduces exercise-induced BDNF and inhibits exercise-related cognitive improvements [93]. Similarly, blocking VEGF reverses running-induced hippocampal neurogenesis [95].

In aged animals (24 months), treadmill running has been shown to increase BDNF and IGF-1 after 4 weeks of intervention, and these changes were associated with improved spatial learning and memory [96]. However, another study in aged animals showed that there was a transient increase in BDNF levels after 1 week of a wheel running that returned to baseline levels in subsequent weeks (2-4 weeks). In this study, it was found that aged animals did not increase their running distance each week whereas the other study utilized a 
treadmill which standardized the exercise exposure, which may account for the transient increase found in voluntary wheel-running. Furthermore, improvements in memory and increases in BDNF have been shown after 8 weeks of aerobic and strength training, showing possible benefits beyond aerobic exercise [97]. Table 4 summarizes the results of studies investigating the effects of exercise on trophic factors in humans.

\section{BDNF}

Three studies that utilized short-term exercise interventions (between 4 and 10 weeks) proposed moderate-load resistance training, either in isolation $[98,99]$ or combined with aerobic exercise [100]. Moderate-load resistance training increased BDNF levels when compared with baseline [99], but not when compared with a control [98]. The combined intervention also did not improve BDNF levels [100]. These studies suggest that short-term exposure to resistance training may not be associated with increases in BDNF.

Many studies assessed the effects of various isolated modes of short-duration (12 or 16 weeks) exercise. None of the following moderate-intensity aerobic activities modulated BDNF levels: walking or running [19] and cycling [51] and light and moderate-load resistance training [101-103]. Four studies employed combined exercise programs that contained resistance training: high-intensity aerobic cycling followed by moderate level coordination/strength [104], high-intensity aerobic cycling followed by moderate level coordination/strength [105], moderate-load resistance training followed by moderate- to high-intensity aerobic training [106], and combined aerobic/strength/coordination [107]. Of these, only the moderate-load resistance training followed by moderate- to high-intensity aerobic training and the combined aerobic/ strength/coordination reported increased BDNF levels, and both reported improvements in short-term memory and processing speed $[106,107]$. Taken together, these studies suggest that medium exposure to aerobic or resistance training delivered in isolation might not be sufficient to influence BDNF levels. In addition, the only 2 studies that demonstrated BDNF increases employed a combined intervention of approximately 2000 total intervention minutes (i.e., $32 \mathrm{~h}$ ), and therefore, the additional load posed by a second intervention and the greater exposure to exercise could have contributed to the results.

Three studies investigated medium-duration exercise (6 months), employing various modes of exercise: highintensity aerobic, moderate-intensity aerobic, and a dancebased intervention $[15,54,108]$. The studies that utilized moderate- or high-intensity aerobic exercise in isolation found no changes in BDNF levels $[15,108]$. Rehfeld et al. [54] found that a dance-based intervention was associated with increased BDNF levels, when compared to an endurance/ strength/flexibility group. The dance intervention required subjects to memorize routines, perform complex choreography, and was regularly changed throughout the study, whereas the comparison group utilized an unchanging exercise regimen, which may have been related to the increases in BDNF found in the intervention group. However, despite the discrepancies in BDNF findings, all studies demonstrated improvements in a range of cognitive abilities (processing speed, visuospatial memory, and episodic memory).

Three studies evaluated the effects of long-duration exercise in a 12-month moderate aerobic walking intervention compared to a stretching and toning group. It appears that these studies are from 1 larger sample, with each study reporting on a subset of the total sample: 92 subjects [21], 120 subjects [14], and 65 subjects [109]. There were no changes in BDNF levels from before to after in the intervention groups in any of the cohorts. A secondary analysis performed by Leckie et al. [21] showed that when dividing the sample by age, younger and older than 65 years of age, the intervention group had an increase in BDNF levels, whereas the control group had a decrease in BDNF levels in individuals older than 65 years old. Both Erickson et al. [14] and Leckie et al. [21] assessed and reported improvements in spatial memory function and task-switching after the intervention. These findings are consistent with the previous results that suggest that only aerobic activity may not be sufficient to modulate BDNF levels. However, it is possible that in individuals over 65 who are susceptible to decreases in BDNF [110], that the prolonged effects of a year-long aerobic intervention may have a positive influence on BDNF levels whereas shorter duration interventions are potentially unable to have the same effect.

\section{IGF and VEGF}

Six studies utilized short-duration exercise (10-12 weeks) of various modes: moderate aerobic exercise [19, 111, 112], combined moderate- and high-intensity aerobic and resistance training $[111,113]$, and low or moderate resistance training $[112,114,115]$. The moderate-intensity aerobic exercise interventions had no effect on IGF-1 levels $[19,111,112]$ or VEGF levels [19]. In 2 studies utilizing combined aerobic and resistance training, 1 found within-group increases in IGF-1, regardless of intervention order [113], whereas the other found no changes when compared to a control group. In the studies utilizing resistance training, only 1 study utilizing moderateintensity exercise found increases in IGF-1 [112], whereas the other 2 found no changes. Both studies that found increased IGF-1 after the intervention (combined or resistance) were in participants in their late $60 \mathrm{~s}$, whereas the studies that found no changes were in people 80 years old $[114,115]$ or 50 years old [111]. These findings may suggest that short exposure to both a combined approach and moderate-intensity resistance 


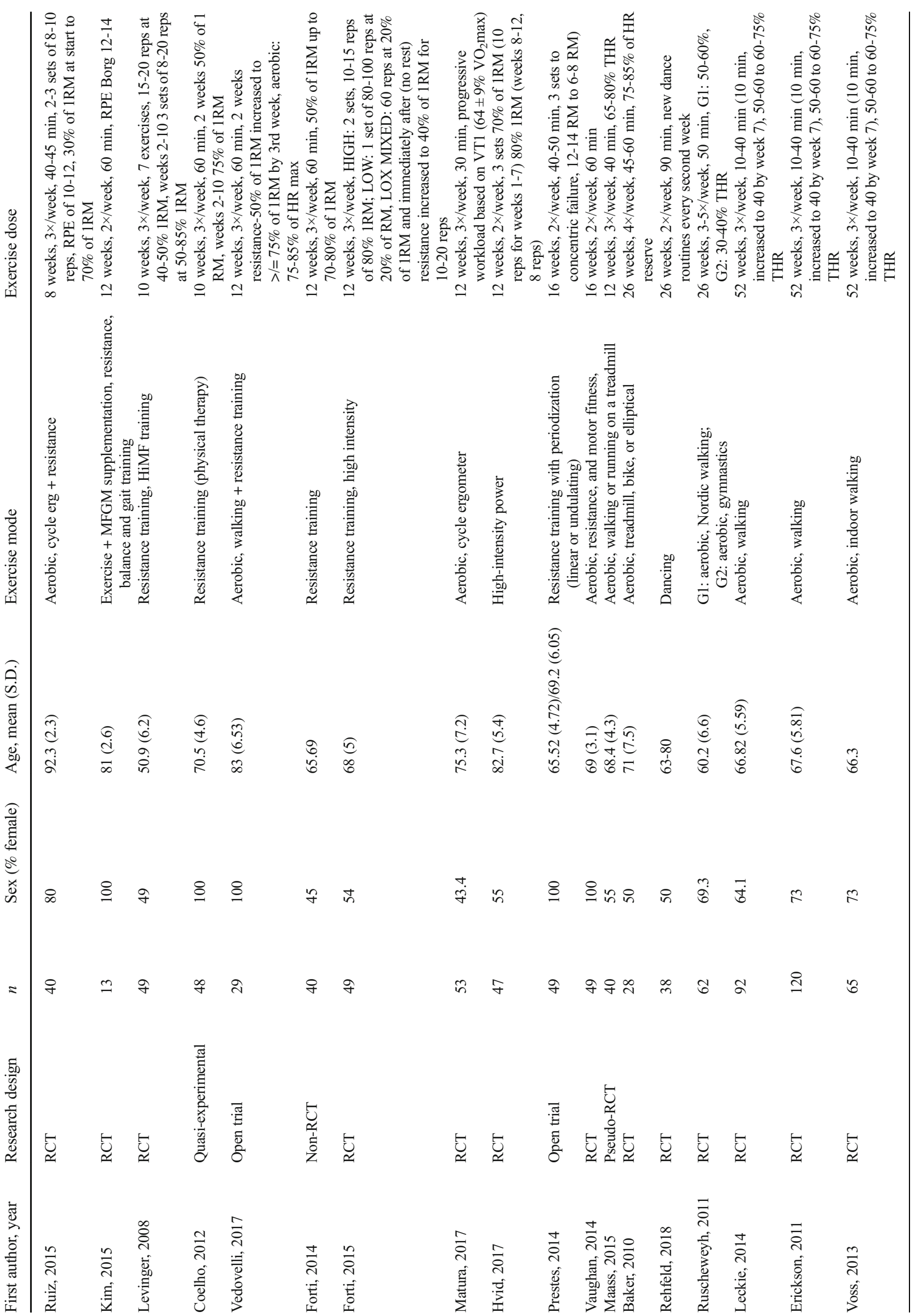




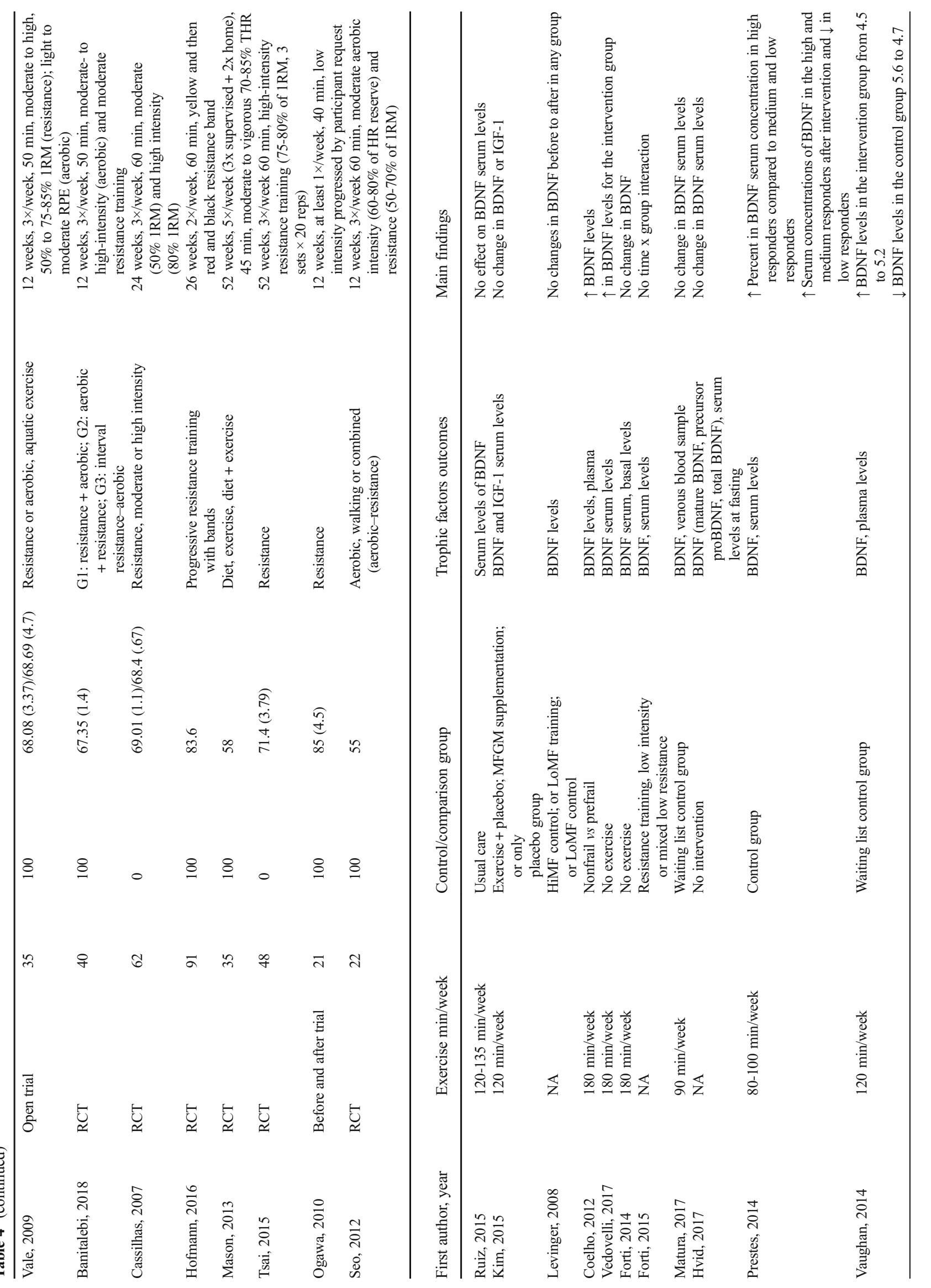




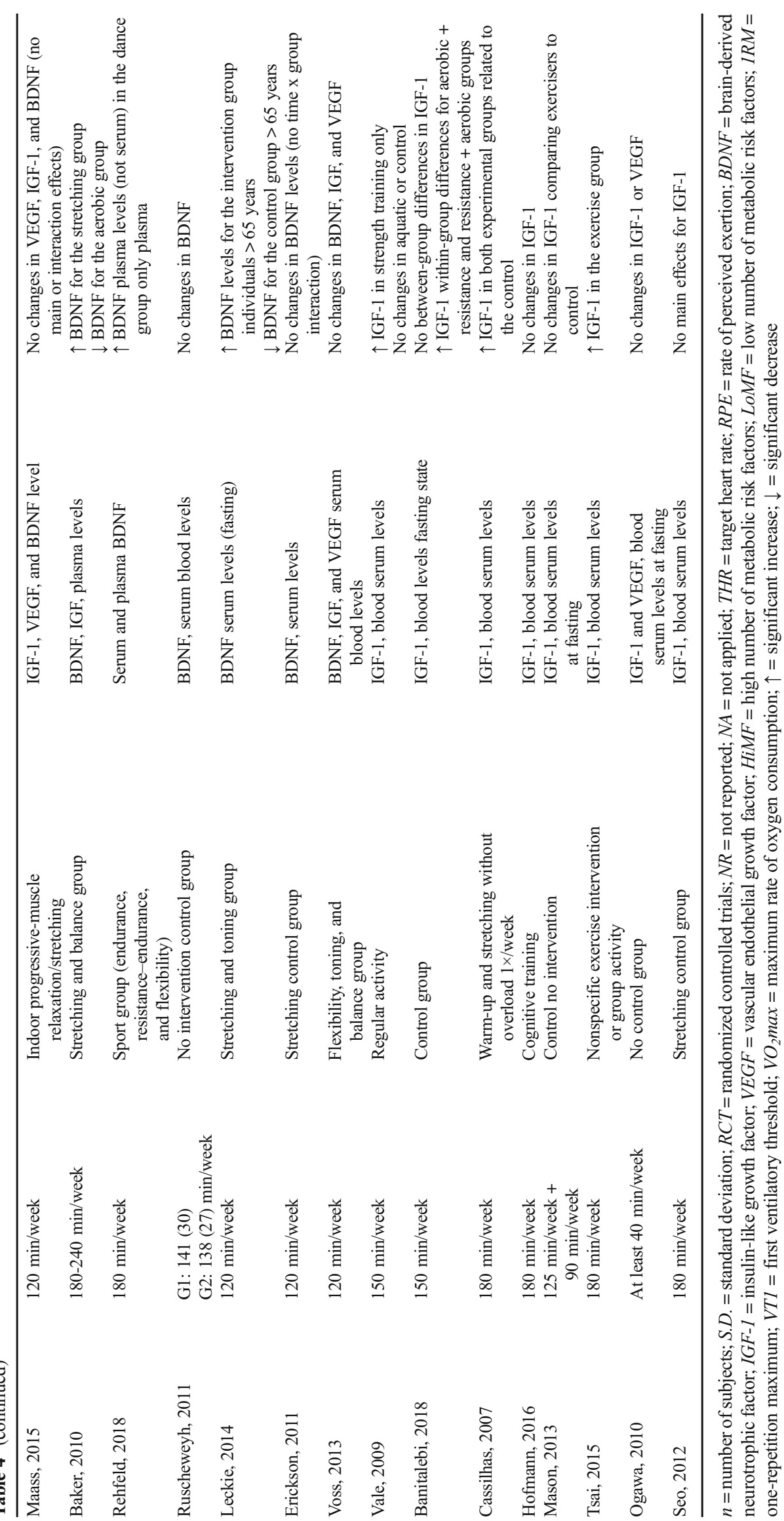


training may increase IGF-1 levels, but there may be an optimal window with regard to age range (at approximately 60 years of age).

Three studies utilized medium-duration exercise (24 weeks) including moderate- to high-intensity aerobic [108] and moderate to high resistance training [116, 117]. The aerobic exercise showed no changes in IGF-1 [108], but the cognitive assessment revealed improvements in executive function (task-switching and visuospatial processing speed). Only 1 of 2 studies found moderate- and high-intensity resistance training to increase IGF-1 levels [116]. A possible reason for the difference in findings is that Cassilhas et al. [116] achieved $72 \mathrm{~h}$ of total intervention compared to $48 \mathrm{~h}$ [117]. Additionally, Cassilhas et al. [116] showed that the intervention group had improvements in visual processing and shortterm memory. As such, in the medium term, resistance training, but not aerobic, associated with memory and attention improvements, possibly due to longer intervention duration, may lead to increases in IGF-1.

Three studies used long-duration exercise (52 weeks), employing moderate to vigorous aerobic [109, 118] and high-load resistance training [119]. Neither aerobic exercise intervention demonstrated a change in IGF-1 when compared to a control or comparison group. Voss et al. [109] also looked at the effects of aerobic intervention on VEGF, finding no changes [109]. High-load resistance training compared to a control group was found to increase IGF-1 and improve processing speed [119]. Overall, these studies provide evidence that short, medium, and long exposure to moderate- to high-intensity resistance training may increase IGF-1 levels in aging adults; however, no consistent effects from aerobic exercise have been evidenced.

\section{The Takeaway: Dose Effects of Exercise in Aging Adults}

In this review, we have summarized the evidence on the effects of different intervals of exposure to exercise (short[1 day-16 weeks], medium- [24-40 weeks], and long-term exercise [52 weeks and beyond]) on the most well-accepted mechanisms used to explain the link between the practice of regular exercise and the improvement in cognitive performance. Due to the heterogeneity in studies, it was not possible to report on domain-specific mechanistic changes. However, evidence from a large-scale systematic review and regression indicates that the most stable and consistent improvements in cognition following exercise occur in executive functions and processing speed [12]. Therefore, we discussed changes in cerebral perfusion, synaptic neuroplasticity, brain structure (volume and connectivity), neurogenesis and synaptogenesis, and trophic factors (BDNF, IGF-1, and VEGF) following participation of exercise in older adults or aged rodents (if no human data was available), that would underlie these improvements in cognitive abilities. We refer to Fig. 1 for a time-guided discussion of the exercise-mediated improvements in mechanisms related to brain health in humans.

\section{Short Term (1 Day to 16 Weeks)}

Even with very short aerobic exercise interventions (i.e., just a few minutes as part of an incremental cycling test), there were increases in resting regional $\mathrm{CBF}$ and $\mathrm{MCA}$ velocity. Short-duration aerobic was the only exercise mode found to be effective at changing connectivity, primarily increasing neural efficiency in frontal and temporal areas, relevant to the processing of cognitive information. The finding of increased connectivity allied to increased brain perfusion is consistent with animal studies that have found that increased connectivity is among the first of exercise-mediated improvements at the brain level, which in turn promotes an increase in angiogenesis to support increased metabolism, ultimately leading to neurogenesis $[76,79]$. The only other structural improvement also associated with aerobic exercise was increased hippocampal volume, which was associated with increased cerebral blood flow to the hippocampus. Despite all of the aforementioned beneficial structural brain changes, aerobic exercise did not seem to change neurobiological factors, as increases in BDNF and IGF-1 were only found with resistance training or combined aerobic and resistance training. Therefore, for the most global benefits from exercise, a combined approach utilizing both aerobic and resistance exercises with at least moderate intensity will contribute best to improved brain structure, cerebral blood flow, as well as improvements in neurobiological factors such as BDNF and IGF-1, in the short-term.

\section{Medium Term (24 to 40 Weeks)}

As the intervention time increased to medium exposure, there continued to be findings of increased connectivity with aerobic exercise. In addition, there were more consistent and diverse structural changes found in terms of increased white and gray matter and volumetric increases mainly in the in frontal and temporal areas (such as the hippocampus, cingulate, and frontal cortices). BDNF was only increased when individuals engaged in an aerobic dance intervention and IGF-1 was only increased when individuals engaged in moderate to high resistance training. We also found some patterns in the studies and identified 3 isolated characteristics that seemed to be associated with the greater likelihood of finding a benefit: including a progressive intervention that increased the exercise intensity from moderate to high, an exercise intervention with at least 150 weekly minutes, and constant novelty (a choreography or the combination of exercise modes). 


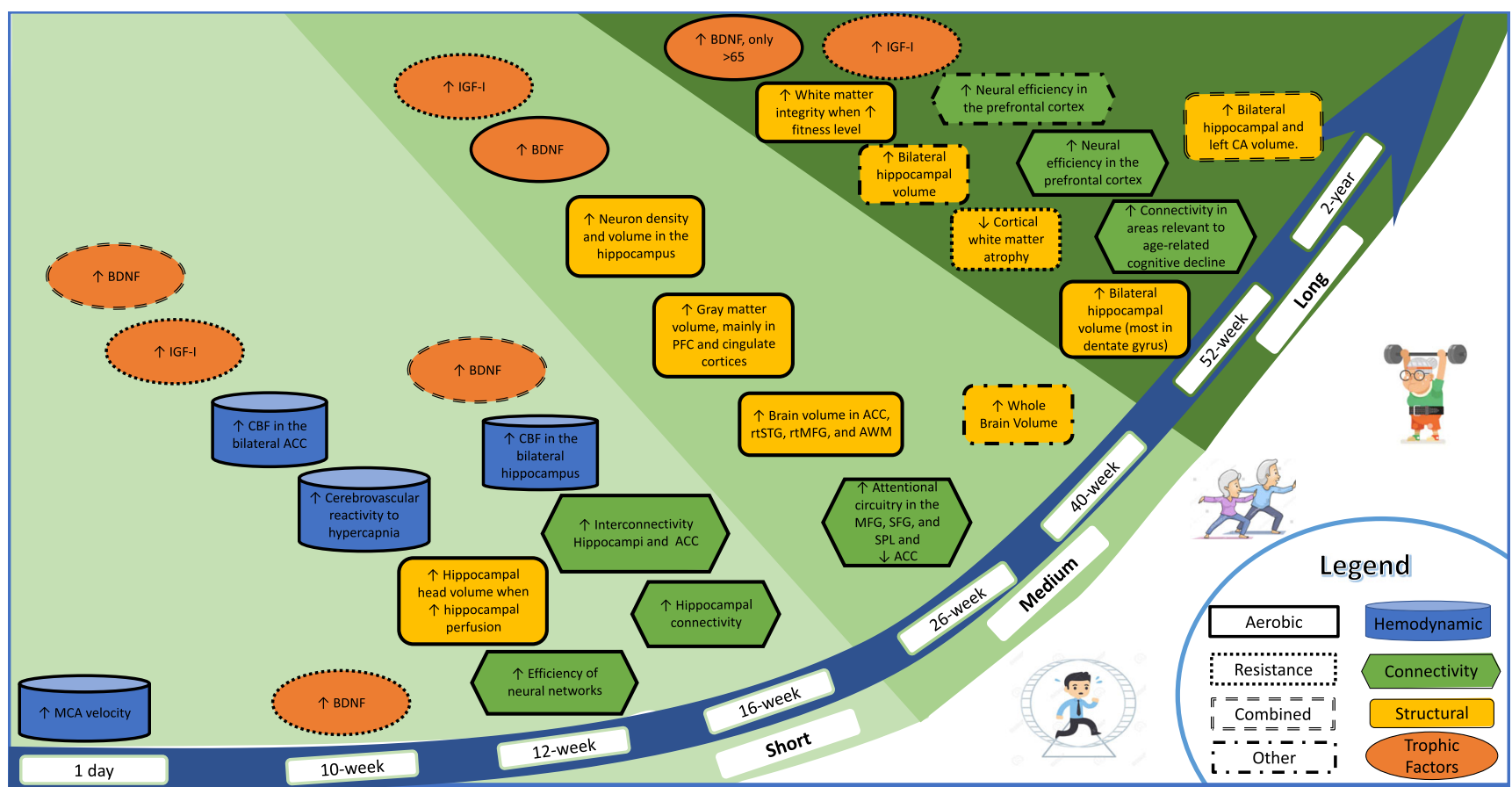

Fig. 1 The significant changes in cerebral perfusion, brain structure, and connectivity and trophic factors with short-, medium-, and long-term exercise. The findings are characterized by the type of exercise performed: aerobic, resistance, combined, or other (i.e., tai chi). $\mathrm{MCA}=$ middle cerebral artery; $\mathrm{CBF}=$ cerebral blood flow; $\mathrm{BDNF}=$ brain-derived neurotrophic factor; IGF-1 = insulin-like growth factor-1; VEGF =

Although the present review synthesizes results from healthy aging individuals, it is pertinent to also consider the role of commonly seen cardiovascular risk factors on the baseline level of risk for cognitive decline. Cardiovascular risk factors are also risk factors for cognitive impairment [120]. Hypertension (present in $65 \%$ of adults older than 60) [121], diabetes mellitus (present in $25 \%$ of adults older than 65 ) [122], obesity (present in $40 \%$ of adults older than 60), smoking (present in $8 \%$ of adults 65 and over), and insufficient activity (present in $60 \%$ of adults older than 65) are all associated with poor cognitive health [123]. Notably, agerelated cognitive changes associated with vascular risk factors are likely mediated through small vessel disease, including white matter pathology. For example, in a sample of 113 aging adults, $67 \%$ of individuals who had white matter lesions also had cardiovascular diseases, which in turn was linked to greater impaired visual functions, mental flexibility, and attention [120]. Favorably however, maintaining a regular exercise regimen for approximately 3 to 6 months can improve maximal aerobic capacity (the gold standard for cardiorespiratory fitness) in older adults [124]. The increased cardiovascular fitness can have a role in modifying these risk factors, thus contributing to potential improvements in cognitive brain health and, additionally, enlisting neurohumoral processes controlling the cardiovascular and endothelial systems $[123,125]$. vascular endothelial growth factor; $\mathrm{ACC}=$ anterior cingulate cortex; $\mathrm{PFC}=$ prefrontal cortex; rtSTG = right superior temporal gyrus; rtMFG = right medial frontal gyrus; $\mathrm{AWM}=$ anterior white matter; $\mathrm{MFG}=$ medial frontal gyrus; $\mathrm{SFG}=$ superior frontal gyrus; $\mathrm{SPL}=$ superior parietal lobules; $\mathrm{CA}=$ cornus ammonis

\section{Long Term (52 Weeks and Beyond)}

Studies in long-term exercise included exercise modes beyond aerobic interventions. Aerobic, coordination, and combined exercise interventions were linked to increases in white matter integrity and increased hippocampal volume, which seemed to be driven by an improvement in cardiovascular fitness through moderate to high exercise intensities. In addition, these interventions were associated with increased neural efficiency. Resistance training was shown to be effective at increasing IGF-1. These studies have also provided evidence that different exercise modes exert benefits via distinct mechanisms, which further supports the suggestion that engaging in physical exercise of different modes will lead to the greatest benefits on brain health.

Acknowledgments Dr. Gomes-Osman was supported by an Evelyn F. McKnight Pilot Grant. The project described was supported by the National Center for Advancing Translational Sciences of the National Institutes of Health under Award Number KL2TR002737. The content is solely the responsibility of the authors and does not necessarily represent the official views of the National Institutes of Health.

Required Author Forms Disclosure forms provided by the authors are available with the online version of this article. 


\section{References}

1. Bureau UC. An aging nation: projected number of children and older adults. Available at: https://www.census.gov/library/ visualizations/2018/comm/historic-first.html. Accessed February 2019.

2. Blazer DG, Yaffe K, Karlawish J. Cognitive aging. JAMA 2015;313:2121.

3. DeCarli C, Massaro J, Harvey D, et al. Measures of brain morphology and infarction in the framingham heart study: establishing what is normal. Neurobiol Aging 2005;26:491-510.

4. Raz N, Gunning-Dixon F, Head D, et al. Aging, sexual dimorphism, and hemispheric asymmetry of the cerebral cortex: replicability of regional differences in volume. Neurobiol Aging 2004;25:377-396.

5. Dong C, Nabizadeh N, Caunca M, et al. Cognitive correlates of white matter lesion load and brain atrophy: the Northern Manhattan Study. Neurology 2015;85:441-449.

6. Mattson MP, Maudsley S, Martin B. BDNF and 5-HT: a dynamic duo in age-related neuronal plasticity and neurodegenerative disorders. Trends Neurosci 2004;27:589-594.

7. Mukherjee J, Christian BT, Dunigan KA, et al. Brain imaging of $18 \mathrm{~F}$-fallypride in normal volunteers: blood analysis, distribution, test-retest studies, and preliminary assessment of sensitivity to aging effects on dopamine D-2/D-3 receptors. Synapse 2002;46: 170-188.

8. Nyberg L. Intact frontal memory effect in older age and dementia. Neuron 2004;42:701-2.

9. Hsieh H, Boehm J, Sato C, et al. AMPAR removal underlies abeta-induced synaptic depression and dendritic spine loss. Neuron 2006;52:831-843.

10. Lobelo F, Stoutenberg M, Hutber A. The exercise is medicine global health initiative: a 2014 update. Br J Sport Med . 2014;48:1627-1633.

11. Piercy KL, Troiano RP, Ballard RM, et al. The physical activity guidelines for Americans. JAMA - J Am Med Assoc 2018;320: 2020-2028.

12. Gomes-Osman J, Cabral DF, Morris TP, et al. Exercise for cognitive brain health in aging. Neurol Clin Pract 2018;1-9.

13. Colcombe SJ, Erickson KI, Scalf PE, et al. Aerobic exercise training increases brain volume in aging humans. J Gerontol Ser A Biol Sci Med Sci 2006;61:1166-1170.

14. Erickson KI, Voss MW, Prakash RS, et al. Exercise training increases size of hippocampus and improves memory. Proc Natl Acad Sci 2011;108:3017-3022.

15. Ruscheweyh R, Willemer C, Krüger K, et al. Physical activity and memory functions: an interventional study. Neurobiol Aging 2011;32:1304-1319.

16. Weinstein AM, Voss MW, Prakash RS, et al. The association between aerobic fitness and executive function is mediated by prefrontal cortex volume. Brain Behav Immun 2012;26:811-819.

17. Maass A, Duzel S, Goerke M, et al. Vascular hippocampal plasticity after aerobic exercise in older adults. Mol Psychiatry 2015;20:585-593.

18. Christie BR, Eadie BD, Kannangara TS, Robillard JM, Shin J, Titterness AK. Exercising our brains: how physical activity impacts synaptic plasticity in the dentate gyrus. NeuroMolecular Med 2008 Jun;10:47-58.

19. Maass A, Düzel S, Brigadski T, et al. Relationships of peripheral IGF-1, VEGF and BDNF levels to exercise-related changes in memory, hippocampal perfusion and volumes in older adults. Neuroimage 2016;131:142-154.

20. Knaepen K, Goekint M, EM H, Meeusen R. Neuroplasticityexercise-induced response of peripheral brain-derived neurotrophic factor: a systematic review of experimental studies in human subjects. Sport Med 2010;40:765-801.

21. Leckie RL, Oberlin LE, Voss MW, et al. BDNF mediates improvements in executive function following a 1-year exercise intervention. Front Hum Neurosci 2014;8:985.

22. Rasmussen $\mathrm{P}$, Brassard $\mathrm{P}$, Adser $\mathrm{H}$, et al. Evidence for a release of brain-derived neurotrophic factor from the brain during exercise. Exp Physiol 2009;94:1062-1069.

23. Cotman CW, Berchtold NC, Christie LA. Exercise builds brain health: key roles of growth factor cascades and inflammation. Trends Neurosci 2007;30:464-472.

24. Erickson KI, Hillman CH, Kramer AF. Physical activity, brain, and cognition. Curr Opin Behav Sci 2015;4:27-32.

25. Chieffi S, Messina G, Villano I, et al. Neuroprotective effects of physical activity: evidence from human and animal studies. Front Neurol 2017;8:1-7.

26. Stimpson NJ, Davison G, Javadi AH. Joggin' the noggin: towards a physiological understanding of exercise-induced cognitive benefits. Neurosci Biobehav Rev 2018;88:177-86.

27. Schneider WJ, McGrew KS. The Cattell-Horn-Carroll model of intellidence. In: DP Flanagan and PL Harrison, editor. Contemporary intellectual assessment theories, tests and issues. 3rd ed New York: Guilford; 2012. p. 99-138.

28. Buijs PC, Krabbe-Hartkamp MJ, Bakker CJ, et al. Effect of age on cerebral blood flow: measurement with ungated two-dimensional phase-contrast MR angiography in 250 adults. Radiology 1998;209:667-674.

29. Tarumi T, Ayaz Khan M, Liu J, et al. Cerebral hemodynamics in normal aging: central artery stiffness, wave reflection, and pressure pulsatility. J Cereb Blood Flow Metab 2014;34:971-978.

30. Moraine JJ, Lamotte M, Berré J, Niset G, Leduc A, Naeije R. Physiology and occupational physiology relationship of middle cerebral artery blood flow velocity to intensity during dynamic exercise in normal subjects. Eur J Appl Physiol 1993;67:35-38.

31. Fisher JP, Hartwich D, Seifert T, et al. Cerebral perfusion, oxygenation and metabolism during exercise in young and elderly individuals. J Physiol 2013;591:1859-1870.

32. Flück D, Braz ID, Keiser S, et al. Age, aerobic fitness, and cerebral perfusion during exercise: role of carbon dioxide. Am J Physiol Circ Physiol 2014;307:515-523.

33. Murrell CJ, Cotter JD, Thomas KN, Lucas SJE, Williams MJA, Ainslie PN. Cerebral blood flow and cerebrovascular reactivity at rest and during sub-maximal exercise: effect of age and 12-week exercise training. Age (Omaha) 2013;35:905-920.

34. Chapman SB, Aslan S, Spence JS, et al. Shorter term aerobic exercise improves brain, cognition, and cardiovascular fitness in aging. Front Aging Neurosci 2013;5:1-9.

35. Chapman SB, Aslan S, Spence JS, et al. Distinct brain and behavioral benefits from cognitive vs. physical training: a randomized trial in aging adults. Front Hum Neurosci. 2016;10:1-15.

36. Burdette JH, Laurienti PJ, Espeland MA, et al. Using network science to evaluate exercise-associated brain changes in older adults. Front Aging Neurosci 2010;2:1-10.

37. Bliss TVP, Collingridge GL. A synaptic model of memory: longterm potentiation in the hippocampus. 1993.

38. Hölscher C. Synaptic plasticity and learning and memory: LTP and beyond. J Neurosci Res 1999;58:62-75.

39. Bliss TVP, Gardner-Medwin AR. Long-lasting potentiation of synaptic transmission in the dentate area of the unanaesthetized rabbit following stimulation of the perforant path. J Physiol 1973;232.

40. Van Praag H. Neurogenesis and exercise: past and future directions. NeuroMolecular Med 2008;10:128-140.

41. Christie BR, Swann SE, Fox CJ, et al. Voluntary exercise rescues deficits in spatial memory and long-term potentiation in prenatal ethanol-exposed male rats. Eur J Neurosci 2005;21:1719-1726. 
42. Zhao G, Liu HL, Zhang H, Tong AXJ. Treadmill exercise enhances synaptic plasticity, but does not alter $\hat{\mathrm{I}}^{2}$-amyloid deposition in hippocampi of aged APP/PS1 transgenic mice. Neuroscience 2015;298:357-66.

43. Liu H-L, Zhao G, Cai K, Zhao H-H, Shi L-D. Treadmill exercise prevents decline in spatial learning and memory in APP/PS1 transgenic mice through improvement of hippocampal long-term potentiation. Behav Brain Res 2010;218:308-14.

44. Dao AT, Zagaar MA, Salim S, Eriksen JL, Alkadhi KA. Regular exercise prevents non-cognitive disturbances in a rat model of Alzheimer's disease. Int J Neuropsychopharmacol 2014;17:593602 .

45. Kumar A, Rani A, Tchigranova O, Lee W, Foster TC. Influence of late-life exposure to environmental enrichment or exercise on hippocampal function and CA1 senescent physiology. Neurobiol Aging. 2012;33:828.e1-828.e17.

46. O'Callaghan RM, Griffin ÉW, Kelly ÁM. Long-term treadmill exposure protects against age-related neurodegenerative change in the rat hippocampus. Hippocampus 2009;19:1019-1029.

47. Raz N, Lindenberger U, Rodrigue KM, et al. Regional brain changes in aging healthy adults: general trends, individual differences and modifiers. Cereb Cortex . 2005;15:1676-1689.

48. Bugg JM, Head D. Exercise moderates age-related atrophy of the medial temporal lobe. Neurobiol Aging . 2011;32:506-514.

49. Colcombe SJ, Erickson KI, Raz N, et al. Aerobic fitness reduces brain tissue loss in aging humans. Journals Gerontol Ser A Biol Sci Med Sci . 2003;58:M176-M180.

50. Carlson MC, Xue QL, Zhou J, Fried LP. Executive decline and dysfunction precedes declines in memory: the Women's Health and Aging Study II. J Gerontol - Ser A Biol Sci Med Sci 2009;64:110-117.

51. Matura S, Fleckenstein J, Deichmann R, et al. Effects of aerobic exercise on brain metabolism and grey matter volume in older adults: results of the randomised controlled SMART trial. Transl Psychiatry 2017;7:e1172.

52. Kleemeyer MM, Kühn S, Prindle J, et al. Changes in fitness are associated with changes in hippocampal microstructure and hippocampal volume among older adults. Neuroimage . 2016;131: $155-161$.

53. Jonasson LS, Nyberg L, Kramer AF, Lundquist A, Riklund K, Boraxbekk C-J. Aerobic exercise intervention, cognitive performance, and brain structure: results from the Physical Influences on Brain in Aging (PHIBRA) study. Front Aging Neurosci. 2017;8: $1-15$.

54. Rehfeld K, Lüders A, Hökelmann A, et al. Dance training is superior to repetitive physical exercise in inducing brain plasticity in the elderly. Buchowski MS, editor. PLoS One 2018;13:e196636.

55. Mortimer JA, Ding D, Borenstein AR, et al. Changes in brain volume and cognition in a randomized trial of exercise and social interaction in a community-based sample of non-demented Chinese elders. J Alzheimers Dis . 2012;30:757-766.

56. Liu-Ambrose T. Resistance training and executive functions. Arch Intern Med . 2010;170:170.

57. Enzinger C, Fazekas F, Matthews PM, et al. Risk factors for progression of brain atrophy in aging: six-year follow-up of normal subjects. Neurology 2005;64:1704-1711.

58. Fotenos AF, Snyder AZ, Girton LE, Morris JC, Buckner RL. Normative estimates of cross-sectional and longitudinal brain volume decline in aging and AD. Neurology . 2005;64:1032-1039.

59. Best JR, Chiu BK, Liang Hsu C, Nagamatsu LS, Liu-Ambrose T. Long-term effects of resistance exercise training on cognition and brain volume in older women: results from a randomized controlled trial. J Int Neuropsychol Soc 2015;21:745-756.

60. Voss MW, Heo S, Prakash RS, et al. The influence of aerobic fitness on cerebral white matter integrity and cognitive function in older adults: results of a one-year exercise intervention. Hum Brain Mapp 2012;34:2972-2985.

61. Niemann C, Godde B, Voelcker-Rehage C. Not only cardiovascular, but also coordinative exercise increases hippocampal volume in older adults. Front Aging Neurosci 2014;6:1-24.

62. Burgess N, Maguire EA, O'Keefe J. The human hippocampus and spatial and episodic memory. Neuron 2002;35:625-641.

63. Rosano C, Guralnik J, Pahor M, et al. Hippocampal response to a 24-month physical activity intervention in sedentary older adults. Am J Geriatr Psychiatry 2017;25:209-217.

64. Thomas C, Moya L, Avidan G, et al. Reduction in white matter connectivity, revealed by diffusion tensor imaging, may account for age-related changes in face perception. J Cogn Neurosci 2008;20:268-284.

65. Grady CL, McIntosh AR, Craik FIM. Age-related differences in the functional connectivity of the hippocampus during memory encoding. Hippocampus 2003;13:572-586.

66. Dennis NA, Hayes SM, Prince SE, Madden DJ, Huettel SA, Cabeza R. Effects of aging on the neural correlates of successful item and source memory encoding. J Exp Psychol Learn Mem Cogn 2008;34:791-808.

67. Pudas S, Josefsson M, Rieckmann A, Nyberg L. Longitudinal evidence for increased functional response in frontal cortex for older adults with hippocampal atrophy and memory decline. Cereb Cortex 2017;936-948.

68. Smith JC, Nielson K A, Woodard JL, Seidenberg M, Rao SM. Physical activity and brain function in older adults at increased risk for Alzheimer's disease. Brain Sci 2013;3:54-83.

69. Stevens FL, Hurley RA, Taber KH. Anterior cingulate cortex: unique role in cognition and emotion. J Neuropsychiatry Clin Neurosci 2011;23:121-125.

70. Colcombe SJ, Kramer AF, Erickson KI, et al. Cardiovascular fitness, cortical plasticity, and aging. Proc Natl Acad Sci 2004;101: 3316-3321.

71. Voss MW, Prakash RS, Erickson KI, et al. Plasticity of brain networks in a randomized intervention trial of exercise training in older adults. Front Aging Neurosci 2010;2:1-17.

72. Buckner RL, Andrews-Hanna JR, Schacter DL. The brain's default network. Ann N Y Acad Sci 2008;1124:1-38.

73. Dosenbach NUF, Visscher KM, Palmer ED, et al. A core system for the implementation of task sets. Neuron 2006;50:799-812.

74. Voelcker-Rehage C, Godde B, Staudinger UM. Cardiovascular and coordination training differentially improve cognitive performance and neural processing in older adults. Front Hum Neurosci 2011;5:1-12.

75. van Praag H, Kempermann G, Gage FH. Running increases cell proliferation and neurogenesis in the adult mouse dentate gyrus. Nat Neurosci 1999;2:266-270.

76. Pereira AC, Huddleston DE, Brickman AM, et al. An in vivo correlate of exercise-induced neurogenesis in the adult dentate gyrus. PNAS 2007;104.

77. Huang Y-Q, Wu C, He X-F, et al. Effects of voluntary wheelrunning types on hippocampal neurogenesis and spatial cognition in middle-aged mice. Front Cell Neurosci 2018;12:1-9.

78. Kronenberg G, Bick-Sander A, Bunk E, Wolf C, Ehninger D, Kempermann G. Physical exercise prevents age-related decline in precursor cell activity in the mouse dentate gyrus. Neurobiol Aging 2006;27:1505-1513.

79. van Praag H. Exercise enhances learning and hippocampal neurogenesis in aged mice. J Neurosci 2005;25:8680-8685.

80. Gibbons TE, Pence BD, Petr G, et al. Voluntary wheel running, but not a diet containing (-)-epigallocatechin-3-gallate and $\beta$-alanine, improves learning, memory and hippocampal neurogenesis in aged mice. Behav Brain Res . 2014;272:131-140. 
81. Kannangara TS, Lucero MJ, Gil-Mohapel J, et al. Running reduces stress and enhances cell genesis in aged mice. Neurobiol Aging 2011;32:2279-2286.

82. Creer DJ, Romberg C, Saksida LM, van Praag H, Bussey TJ. Running enhances spatial pattern separation in mice. Proc Natl Acad Sci 2010;107:2367-2372.

83. Blackmore DG, Golmohammadi MG, Large B, Waters MJ, Rietze RL. Exercise increases neural stem cell number in a growth hormone-dependent manner, augmenting the regenerative response in aged mice. Stem Cells 2009;27:2044-2052.

84. Itou Y, Nochi R, Kuribayashi H, Saito Y, Hisatsune T. Cholinergic activation of hippocampal neural stem cells in aged dentate gyrus. Hippocampus 2011;21:446-459.

85. Jessop P, Toledo-Rodriguez M. Hippocampal TET1 and TET2 expression and DNA hydroxymethylation are affected by physical exercise in aged mice. Front Cell Dev Biol . 2018;6:1-9.

86. Littlefield AM, Setti SE, Priester C, Kohman RA. Voluntary exercise attenuates LPS-induced reductions in neurogenesis and increases microglia expression of a proneurogenic phenotype in aged mice. J Neuroinflammation 2015;12:1-12.

87. Kohman RA, DeYoung EK, Bhattacharya TK, Peterson LN, Rhodes JS. Wheel running attenuates microglia proliferation and increases expression of a proneurogenic phenotype in the hippocampus of aged mice. Brain Behav Immun 2012;26:803-810.

88. Canas PM, Duarte JMN, Rodrigues RJ, Köfalvi A, Cunha RA. Modification upon aging of the density of presynaptic modulation systems in the hippocampus. Neurobiol Aging 2009;30:1877-1884.

89. Nichol K, Deeny SP, Seif J, Camaclang K, Cotman CW. Exercise improves cognition and hippocampal plasticity in APOE $\varepsilon 4$ mice. Alz Dement 2009;5:287-294.

90. Black JE, Isaacs KR, Anderson BJ, Alcantara AA, Greenough WT. Learning causes synaptogenesis, whereas motor activity causes angiogenesis, in cerebellar cortex of adult rats (paramedian lobule/neural plasticity/exercise). Proc Natl Acad Sci USA 1990;87:5568-5572.

91. Boldrini M, Fulmore CA, Tartt AN, et al. Human hippocampal neurogenesis persists throughout Aging Cell Stem Cell 2018;22: 589-599.e5.

92. Vaynman S, Ying Z, Gomez-Pinilla F. Hippocampal BDNF mediates the efficacy of exercise on synaptic plasticity and cognition. Eur J Neurosci 2004;20:2580-2590.

93. Ding Q, Vaynman S, Akhavan M, Ying Z, Gomez-Pinilla F. Insulin-like growth factor I interfaces with brain-derived neurotrophic factor-mediated synaptic plasticity to modulate aspects of exercise-induced cognitive function. Neuroscience 2006;140: 823-833.

94. Ding Y-H, Li J, Zhou Y, Rafols J, Clark J, Ding Y. Cerebral angiogenesis and expression of angiogenic factors in aging rats after exercise. Curr Neurovasc Res 2006;3:15-23.

95. Fabel K, Fabel K, Tam B, et al. VEGF is necessary for exerciseinduced adult hippocampal neurogensis. Eur J Neurosci 2003;18: 2803-2812.

96. Vanzella C, Neves JD, Vizuete AF, et al. Treadmill running prevents age-related memory deficit and alters neurotrophic factors and oxidative damage in the hippocampus of Wistar rats. Behav Brain Res 2017;334:78-85.

97. Vilela TC, Muller AP, Damiani AP, et al. Strength and aerobic exercises improve spatial memory in aging rats through stimulating distinct neuroplasticity mechanisms. Mol Neurobiol 2017;54: 7928-7937.

98. Levinger I, Goodman C, Matthews V, et al. BDNF, metabolic risk factors, and resistance training in middle-aged individuals. Med Sci Sport Exerc 2008;40:535-541.

99. Coelho FM, Pereira DS, Lustosa LP, et al. Physical therapy intervention (PTI) increases plasma brain-derived neurotrophic factor
(BDNF) levels in non-frail and pre-frail elderly women. Arch Gerontol Geriatr 2012;54:415-420.

100. Ruiz JR, Gil-Bea F, Bustamante-Ara N, et al. Resistance training does not have an effect on cognition or related serum biomarkers in nonagenarians: a randomized controlled trial. Int J Sports Med 2015;36:54-60.

101. Forti LN, Van Roie E, Njemini R, et al. Dose- and gender-specific effects of resistance training on circulating levels of brain derived neurotrophic factor (BDNF) in community-dwelling older adults. Exp Gerontol 2015;70:144-149.

102. Forti LN, Njemini R, Beyer I, et al. Strength training reduces circulating interleukin- 6 but not brain-derived neurotrophic factor in community-dwelling elderly individuals. Age (Omaha) 2014;36:9704.

103. Prestes J, da Cunha Nascimento D, Tibana RA, et al. Understanding the individual responsiveness to resistance training periodization. Age (Omaha) 2015;37:55.

104. Hvid LG, Nielsen MKF, Simonsen C, Andersen M, Caserotti P. Brain-derived neurotrophic factor (BDNF) serum basal levels is not affected by power training in mobility-limited older adults - a randomized controlled trial. Exp Gerontol 2017;93:29-35.

105. Kim H, Suzuki T, Kim M, et al. Effects of exercise and milk fat globule membrane (MFGM) supplementation on body composition, physical function, and hematological parameters in community-dwelling frail Japanese women: a randomized double blind, placebo-controlled, follow-up trial. Buchowski M, editor. PLoS One. 2015;10:e0116256.

106. Vedovelli K, Giacobbo BL, Corrêa MS, Wieck A, Argimon II de L, Bromberg E. Multimodal physical activity increases brainderived neurotrophic factor levels and improves cognition in institutionalized older women. GeroScience 2017;39:407-417.

107. Vaughan S, Wallis M, Polit D, Steele M, Shum D, Morris N. The effects of multimodal exercise on cognitive and physical functioning and brain-derived neurotrophic factor in older women: a randomised controlled trial. Age Ageing 2014;43:623-629.

108. Baker LD, Frank LL, Foster-Schubert K, et al. Aerobic exercise improves cognition for older adults with glucose intolerance, a risk factor for Alzheimer's disease. J Alzheimers Dis 2010;22:569-579.

109. Voss MW, Erickson KI, Prakash RS, Chaddock L, Kim JS, Alves $\mathrm{H}$, et al. Neurobiological markers of exercise-related brain plasticity in older adults. Brain Behav Immun 2013;28:90-99.

110. Lommatzsch M, Virchow JC, Zingler D, et al. The impact of age, weight and gender on BDNF levels in human platelets and plasma. Neurobiol Aging 2004;26:115-123.

111. Seo D-I, Jun T-W, Park K-S, Chang H, So W-Y, Song W. 12 weeks of combined exercise is better than aerobic exercise for increasing growth hormone in middle-aged women. Int J Sport Nutr Exerc Metab 2010;20:21-26.

112. Vale RG, de Oliveira RD, Pernambuco CS, de Meneses YP, Novaes Jda S, Andrade Ade F. Effects of muscle strength and aerobic training on basal serum levels of IGF-1 and cortisol in elderly women Arch Gerontol Geriatr 2009;49:343-7.

113. Banitalebi E, Faramarzi M, Bagheri L, Kazemi AR. Comparison of performing 12 weeks' resistance training before, after and/or in between aerobic exercise on the hormonal status of aged women: a randomized controlled trial. Horm Mol Biol Clin Investig 2018;35:1-10.

114. Ogawa K, Sanada K, MacHida S, Okutsu M, Suzuki K. Resistance exercise training-induced muscle hypertrophy was associated with reduction of inflammatory markers in elderly women. Mediators Inflamm. 2010.

115. Kim H, Suzuki T, Kim M, et al. Effects of exercise and milk fat globule membrane (MFGM) supplementation on body composition, physical function, and hematological parameters in community-dwelling frail Japanese women: a randomized double 
blind, placebo-controlled, follow-up trial. PLoS One 2015;10: $\mathrm{e} 0116256$.

116. Cassilhas RC, Viana VAR, Grassmann V, et al. The impact of resistance exercise on the cognitive function of the elderly. Med Sci Sports Exerc 2007;39:1401-1407.

117. Hofmann M, Schober-Halper B, Oesen S, et al. Effects of elastic band resistance training and nutritional supplementation on muscle quality and circulating muscle growth and degradation factors of institutionalized elderly women: the Vienna Active Ageing Study (VAAS). Eur J Appl Physiol 2016;116:885-897.

118. Mason C, Xiao L, Duggan C, et al. Effects of dietary weight loss and exercise on insulin-like growth factor-i and insulin-like growth factor-binding protein-3 in postmenopausal women: a randomized controlled trial. Cancer Epidemiol Biomarkers Prev 2013;22:1457-1463.

119. Tsai C-L, Wang C-H, Pan C-Y, Chen F-C. The effects of long-term resistance exercise on the relationship between neurocognitive performance and GH, IGF-1, and homocysteine levels in the elderly. Front Behav Neurosci 2015;9:1-12.
120. Ylikoski R, Ylikoski A, Raininko R, et al. Cardiovascular diseases, health status, brain imaging findings and neuropsychological functioning in neurologically healthy elderly individuals. Arch Gerontol Geriatr 2000;30.

121. Nwankwo T, Sug S, Yoon S, Burt V, Gu Q. Data from the National Health and Nutrition Examination Survey. 2011.

122. CDC. National Diabetes Statistics Report, 2017 Estimates of Diabetes and Its Burden in the United States Background. 2017.

123. Gorelick PB, Furie KL, Iadecola C, et al. Defining optimal brain health in adults: a presidential advisory from the American Heart Association/American Stroke Association. Stroke 2017;48.

124. Bouchard C, An P, Rice T, et al. Familial aggregation of VO ( $2 \max )$ response to exercise training: results from the HERITAGE Family Study. J Appl Physiol. 1999;87:1003-1008.

125. Trigiani LJ, Hamel E. An endothelial link between the benefits of physical exercise in dementia. 2017;37:2649-2664.

Publisher's Note Springer Nature remains neutral with regard to jurisdictional claims in published maps and institutional affiliations. 